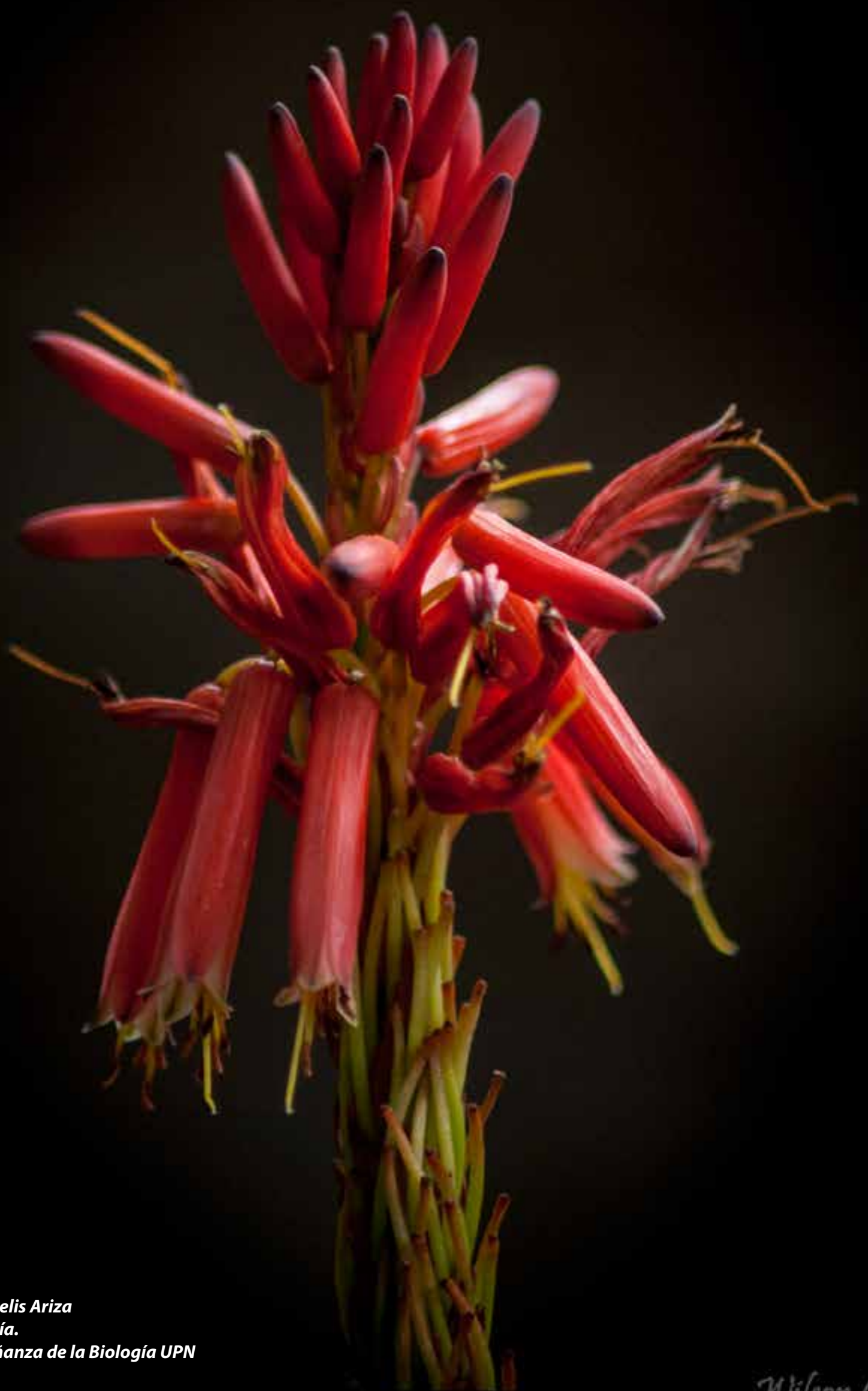

Fotografias Wilson Celis Ariza

Licenciado en Biología.

Especialista en enseñanza de la Biología UPN 


\section{PROGRESIÓN DEL APRENDIZAJE BASADO EN MODELOS: LA ENSEÑANZA YEL APRENDIZAJE DEL SISTEMA NERVIOSO}

\section{Progression of learning based on models: teaching and learning about the nervous system}

Fecha de recepción: 10 de octubre de 2014

Fecha de aprobación: 12 de diciembre de 2014

\section{Resumen}

En esta comunicación presento una propuesta de desarrollo curricular basado en la modelización. Se recuperan las aportaciones de la ciencia escolar en la que se afirma que una de las actividades primordiales de los alumnos es la construcción de modelos teóricos escolares. Los modelos se entienden como tramas de ideas que permiten explicar teóricamente un fenómeno, estas explicaciones se ajustan altamente a las intervenciones experimentales, discursivas y representacionales de los alumnos sobre el mundo. Esta propuesta de desarrollo curricular se basa en la generación de una hipótesis de progresión en el desarrollo de los modelos, asentada en las aportaciones teóricas del área, definiéndose un modelo blanco y cinco modelos intermediarios. Cada modelo intermediario va dirigido a un ciclo educativo, abarcando desde pre-escolar hasta educación secundaria. A partir de la hipótesis de progresión inicial se diseñaron secuencias de aprendizaje, las de pre-escolar y primaria fueron discutidas con los docentes de un jardín de niños y una primaria (10 docentes) y llevadas al aula en condiciones naturales, fueron analizadas y publicadas. A partir del análisis y de la retroalimentación con docentes se reconstruyó la hipótesis de progresión. Consideramos que esta metodología de desarrollo curricular basada en modelización y progresión el aprendizaje, en la cual se retroalimentan teoría y práctica, nos permite generar propuestas de desarrollo para modelos escolares más auténticos, los cuales no consideran únicamente los modelos científicos eruditos, sino las aportaciones del profesorado y los resultados de la práctica escolar con alumnos.

\section{Palabras clave}

Desarrollo curricular, progresión del aprendizaje, modelización.

\author{
Alma Adrianna Gómez Galindo ${ }^{1}$
}

\section{Abstract}

In this paper a proposal for curriculum development modeling based is presented. The contributions of school science were retaken, in this paper a central students' activity is the construction of school theoretical models. The models are understood as structural ideas that allow a theoretical explanation of phenomenon; these explanations are highly consistent with the experimental, discursive and representational activities of the students on the world. This curriculum development is based on the generation of a hypothesis of model progression and uses the theoretical contributions of the area; here or target model and five intermediary models were defining. Each intermediary model is aimed at an educational cycle, ranging from Kindergarten to secondary education. Teaching Learning Sequences were designed, from preschool and primary level, and were discussed with teachers (10 teachers) and performed in the classroom under natural conditions, then they were analyzed and published. From the analysis and feedback from teachers the hypothesized of model progression was rebuilt. We believe that this methodology of model curriculum development, in which a dialogue between theory and practice was used, it allows us to develop more authentic proposals for school models, which not only consider the scientific school models, but the contributions of teachers and students.

\section{Key words}

Curricular development, learning progression, modeling. 


\section{Introducción}

La necesidad de ofrecer un plan de estudios para la enseñanza de la biología en educación básica que sea relevante para el alumnado, pero además accesible considerando los diferentes niveles educativos, es un tema prioritario en el diseño curricular. Un elemento fundamental para guiar el diseño curricular ha de ser el desarrollo gradual de habilidades intelectuales, éticas e interpersonales que permita al estudiantado formar su propia opinión, tomar decisiones y actuar en situaciones relacionadas con el mundo natural y socio-tecnológico. Para lograr lo anterior se requiere la planificación coordinada entre grados y niveles educativos. Diversos estudios han mostrado que los libros de texto para el alumnado presentan una serie de contenidos desvinculados sin coherencia entre niveles educativos y en los que los conceptos relevantes quedan desdibujados (Gómez et al. 2009, Fortus y Krajcik, 2012), lo anterior asociado al hecho de que en la práctica la aplicación real del currículo se lleva a cabo por los profesores utilizando preferentemente materiales instructivos (Lockheed, 1993) resulta relévate, ya que se ha encontrado que la coherencia curricular es uno de los factores predictivos más importantes del desempeño de los alumnos en pruebas como TIMSS (Smith et al., 2005 en Fortus y Krajcik, 2012).

La elaboración de progresiones del aprendizaje es considerada básica para ir de un aprendizaje superficial a una comprensión integrada, en la que las ideas se conectan unas con otras de manera que permiten a los aprendices comprenderlas y usarlas para resolver problemas, plantearse nuevas preguntas y entender el mundo en el que viven (Fortus y Krajcik, 2012). Retomando propuestas socio-constructivistas, estas progresiones de aprendizaje o hipótesis de progresión, se centran en el aprendizaje y no en la enseñanza; hacen referencia al desarrollo gradual, progresivo y evolutivo de un contenido concreto o un conjunto de contenidos conectados entre sí, en el que se van determinando niveles de formulación cuya diferenciación sirve de marco de referencia y de orientación en los procesos dinámicos de enseñanza y aprendizaje (García, 1997, 1998). La coherencia curricular ha sido abordada desde diversas aristas, aquí se integra la modelización como eje vertebrador de una progresión de aprendizaje.

\section{Diseño curricular basado en modelización}

El enfoque basado en modelización es actualmente uno de los principales en los estudios de enseñanza de las ciencias (Sensevy, 2008). La aproximación aquí utilizada proviene de visión semántica a partir de las aportacio- nes del filósofo Ronald Giere (1992), y de la ciencia escolar (Izquierdo et al, 1999) donde se propone que en la ciencia escolar una de las principales actividades de los alumnos es la construcción de modelos científicos escolares. Éstos se entienden como una trama de ideas que permiten explicar teóricamente un fenómeno y que se ajustan altamente a las intervenciones experimentales, discursivas y representacionales de los alumnos sobre el fenómeno estudiado.

A pesar de la polisemia del término, actualmente la modelización en la ciencia escolar se encuentra teóricamente bien fundamentada (Izquierdo et al., 1999, Buckley, 2000, Clement, 2000, Gilbert, 2000, 2002, Tamayo, 2001, Gutiérrez, 2004, Coll et al. 2005, Izquierdo y Adúriz-Bravo, 2003, entre otros). Existen varias propuestas de diseños curriculares a nivel micro, es decir de secuencias de enseñanza aprendizaje en las que se abordan temas específicos (Ver: Van den Akker, 2003, Archer et al. 2007, Gómez et al., 2007). Sin embargo, aún hacen falta diseños curriculares a nivel macro dirigidos a varios grados o niveles educativos, en los que se propongan modelos básicos para construirse paulatinamente a lo largo de la instrucción, señalando cómo estos podrían ir evolucionando a lo largo de la escolaridad.

Una progresión basada en modelización puede recurrir a la propuesta de Clement (2000) en la que presenta un modelo inicial, un modelo blanco y se determinan modelos intermediarios. El modelo blanco sería el que el alumnado podría haber construido al finalizar la educación básica, los modelos intermediarios serían aquellos a desarrollarse en cada ciclo escolar. Tanto los modelos iniciales como intermediarios habrían de ser funcionales en el sentido que permitan explicar un fenómeno e intervenir en él, guiados por ideas teóricas. Sin embrago, también los procesos de modelización han de considerar el desarrollo gradual de habilidades científicas, argumentativas, de evaluación de acciones usando una matriz axiológica, etc.

\section{Objetivos}

En este trabajo pretendo mostrar una propuesta de desarrollo curricular basada en un enfoque de modelización que ha considerado tanto las aportaciones teóricas desarrolladas desde la investigación en el área de didáctica, como las derivadas de los contextos y condiciones de aplicación en el aula. Esta manera de abordar la investigación en la enseñanza de las ciencias genera una mancuerna entre la innovación y la investigación, característica de la didáctica como área de desarrollo (Perales et al. 2002).

La propuesta aquí presentada se concreta en una hipótesis de progresión de los modelos teóricos escolares que 
incluye un modelo blanco y diversos modelos intermediarios para la función relación de lo seres vivos. La sección que aquí se exhibe se centra en las ideas del modelo, sabiendo que las habilidades, capacidades y actitudes han de asociarse a los procesos de modelización de actividades de aula. Concretamente se desarrolla una hipótesis de progresión para abordar el estudio del sistema nervioso a lo largo de un amplio tramo de educación: pre-escolar, primaria y secundaria (de los 5 a los 15 años). El modelo sistema nervioso forma parte de un modelo más amplio, el de "relación", entendida ésta como la capacidad de los seres vivos de recibir y responder a los estímulos del medio y regularse hasta cierto umbral (García 2005).

\section{Desarrollo}

El diseño inicial de las progresión de aprendizaje se basa en resultados de investigaciones antecedentes de investigación, de las cuales se señalan algunas a continuación. Según Sensevy et al. (2008) un elemento central en el desarrollo de modelos es el paso de lo concreto a lo abstracto y viceversa, con la identificación de qué conexiones sistémicas pueden explicar los fenómenos; en este caso, por ejemplo, la capacidad de recibiry responder a los estímulos. Las relaciones entre las entidades abstractas y los fenómenos bajo estudio a su vez se representan usando diversos medios semióticos de forma coordinada (Kress et al. 2001; Gómez, 2008). Se incluye aquí la construcción de entidades, las relaciones entre éstas y sus propiedades, y como éstas se articulan en la producción de explicaciones. Se buscó construir una estructura funcional formal, es decir una forma teórica de pensar, hablar y actuar sobre el mundo.

Respecto al lenguaje, se buscó el desarrollo de nociones compartidas por alumnos, maestros y textos, introducidas paulatinamente, por ejemplo, cerebro, células, fibras nerviosos, umbrales de respuesta, etc. El nuevo lenguaje se asocia a nuevas formas de pensar el fenómeno, identificando las entidades, relaciones y propiedades del modelo, es decir las reglas y conexiones sistémicas del modelo. Se trata de apoyar el desarrollo bidireccional de lo concreto a lo abstracto: la construcción de modelos generan una forma de pensar el mundo pero, al mismo tiempo, una nueva forma de percibirlo y de hablar sobre él.

\section{Metodología}

Inicialmente se realizó una revisión de la propuesta de los libros de texto (Gómez et al. 2009). Se identificaron discontinuidades en el abordaje del tema de sistema nervioso en educación básica, y se busco evitar reproducirlas en la propuesta planteada. Por ejemplo, un aspecto que se consideró fue establecer claras relaciones entre órga- nos de los sentidos y sistema nervioso, aspectos tratados como temas aislados en la propuesta curricular vigente. Posteriormente se realizó el diseño del modelo blanco y de niveles de concresión para cinco modelos intermediaios: 1. pre-escolar (4 y 5 años), 2. primero y segundo años de primaria (6 7 años), 3. tercero y cuarto años de primaria (8 y 9 años) 4 . quinto y sexto años de primaria (10 y 11 años), y 5. primero de secundaria (12 años) ${ }^{2}$. Para cada modelo intermediario se desarrollo una propuesta de secuencia de aprendizaje basada en modelización. Algunas ideas básicas del modelo blanco se presentan en la tabla 1.

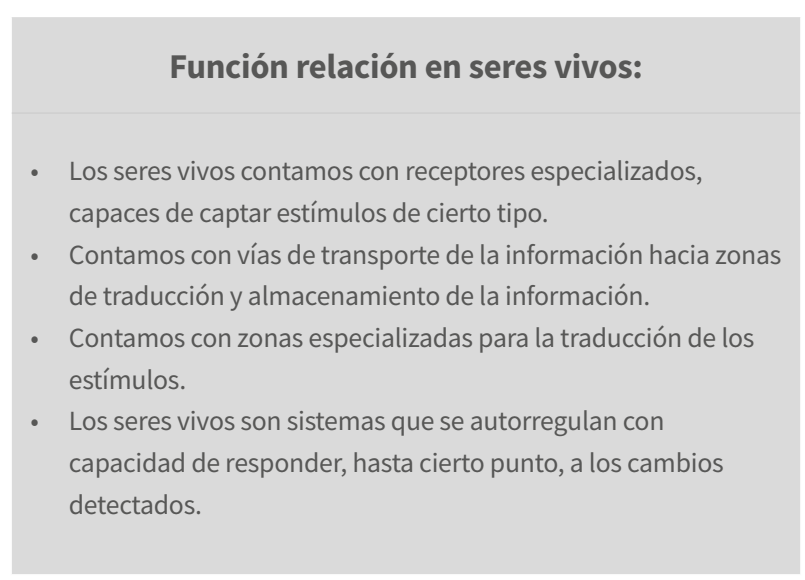

Cuadro1. Algunas ideas básicas del modelo blanco "relación" en seres vivos.

Las ideas del modelo blanco, según la revisión de libros de texto llevada a cabo, se abordan en temas relacionados con órganos de los sentidos y sistema nervioso. Al revisar los programas de pre-escolary primaria, encontramos que en el de preescolar de la Secretaría de Educación Pública (2004) no se mencionan contenidos específicos. Por otra parte, en libro de Planes y Programas de Estudio de la de Primaria (SEP, 1993), se presentan temas relacionados con órganos de los sentidos y sistema nervioso en el primer, cuarto, quinto, y sexto grados.

Posteriormente, en el contexto de un curso sobre modelización dirigido a profesores de una misma escuela, al cual asistieron 10 docentes de educación pre-escolar y primaria, se discutió la propuesta de modelos intermediarios. Tras las observaciones realizadas por los docentes se reformuló la hipótesis de progresión. Realizamos un primer diseño de actividades de aula en forma de secuencia

2 En la escuela secundaría en México, únicamente se estudia biología en el primer año, en el segundo y tercer año se estudian química y física, por ello en esta investigación el modelo ser vivo y la función relación solo se abordaron en primer año de secundaría. 
didáctica, posteriormente este diseño fue comentado con una profesora titular de cada grupo al que la secuencia iba dirigida y se incorporaron las observaciones de las maestras.

Cada Secuencia de actividades retomo la propuesta de Sanmartí (2002) y consistió de aproximadamente siete sesiones de la clase de una hora de duración. Una descripción del tipo de actividades realizadas en cada sesión aparece en el cuadro 2. Las secuencias diseñadas pueden consultarse en Gómez (2009b).

\begin{tabular}{|c|c|}
\hline Sesión & \multicolumn{1}{c|}{ Tipo de actividad } \\
\hline 1 & $\begin{array}{l}\text { Exploración de ideas de partida y comunicación } \\
\text { de objetivos }\end{array}$ \\
\hline 2,3, & $\begin{array}{l}\text { Introducción de nuevos puntos de vista. Rea- } \\
\text { lización de experimentos, uso de analogías, } \\
\text { elaboración de dibujos y maquetas, discusión } \\
\text { grupal y en equipos. }\end{array}$ \\
\hline 6,5 & Síntesis ¿Qué hemos aprendido? \\
\hline
\end{tabular}

Cuadro 2. Actividades generales de las secuencias didácticas diseñadas.

Cada secuencia de actividades se implementó en al menos un grupo-clase en la escuela. En la implementación participaron las profesoras regulares de cada grupo, quienes habían asistido al curso sobre "modelización" y participado en la discusión sobre las actividades a implementar y la persona que escribe estas líneas. Durante las actividades se grabaron las conversaciones, se recogieron los trabajos de los alumnos y se llevó un diario del docente. Los datos anteriores se utilizaron para realizar un análisis cualitativo de las explicaciones construidas por los alumnos, identificando las ideas expresadas o plasmadas en dibujos y maquetas que permitían una explicación teórica del modelo (ver Gómez 2009 a y b, 2011 y 2013). Posteriormente se redefinió la hipótesis de progresión y se re-diseñaron las actividades.

\section{Resultados}

El diseño de la propuesta para la modelización de la función relación que se llevó al aula en el Estado de México y en el Estado de Nuevo León, México. En el nivel de preescolar se realizó en el Jardín de Niños "Niño Eugenio Canavati” en San Nicolás de los Garza, Nuevo León y en el "Colegio Rébsamen”, Naucalpan, Estado de México. Las actividades diseñadas para primaria se llevaron a cabo en la escuela "Carlos Maldonado" Apodaca, Nuevo León, y "Colegio Rébsamen”, Naucalpan, Estado de México.

Tomando el modelo blanco como guía se generaron los cinco modelos intermediarios, los cuales se enriquecieron con los ajustes a las actividades de aula, en los que se consideraron:

- Las aportaciones de los docentes. Por ejemplo en la secuencia Uno (pre-escolar), éstos consideraron que debían trabajarse los tipos de estímulos captados por cada sentido y no hablar de todos juntos como se había planteado inicialmente.

- El ajuste a las actividades como resultado de las reflexiones plasmadas en el diario del docente. Por ejemplo en la secuencia Uno se identificó la importancia de incorporar la idea de que el "cerebro manda órdenes al cuerpo para que se mueva".

- Los resultados del análisis de las explicaciones construidas, incorporando por ejemplo las entidades representadas por los alumnos. El uso del lenguaje es enriquecido por las aportaciones de los alumnos, lo cual nos lleva al re-plantemiento de la forma en que pueden ser expresadas algunas de las ideas teóricas. Por ejemplo en la secuencia Uno la entidad "cablecitos" para expresar la forma en que el cerebro y los sentidos se comunican, priorizandola sobre "tubitos" también usada por el alumnado inicialmente.

La evolución de los modelos se basó en la incorporación de más entidades, propiedades y relaciones en cada modelo intermediario y en la explicación de nuevos fenómenos. Una vez re-elaborados los modelos intermediarios considerando los tres aspectos anteriores, se reformuló el modelo blanco. Con base en lo anterior se generó una segunda hipótesis de progresión, que puede observarse en el cuadro 3. 


\section{Modelo intermediario 1, pre-escolar}

- En nuestro cuerpo tenemos partes especializadas para captar lo que sucede a nuestro alrededor: los órganos de los sentidos (OS).

- Cada parte capta cosas diferentes: la luz (ojos), la temperatura y cuando te toca algo (piel y lengua), el sonido (oídos), el sabor (lengua) y el olor (nariz).

- Los OS se comunican con el cerebro por medio de "cablecitos".

- El cerebro nos dice qué sucede en el ambiente, almacena alguna información y manda ordenes al cuerpo por medio de los "cablecitos" para que reaccione a lo que sucede a su alrededor.

- El cerebro controla los movimientos.

\section{Modelo intermediario 2, primero y segundo años}

- "Otros seres vivos (animales) tienen partes especiales para captar lo que sucede a nuestro alrededor: los órganos de los sentidos"

- "Cada parte especializada capta cosas diferentes: la luz (ojos), la temperatura y cuando les toca algo (piel y lengua), el sonido (oídos), el sabor (lengua) y el olor (nariz)".

- "Los órganos de los sentidos se comunican con el cerebro por medio de cablecitos".

- "Por los cablecitos viaja información que permite a los seres vivos saber lo que sucede a su alrededor"

- "El cerebro de los animales les dice qué sucede en el ambiente, almacena las ideas y manda ordenes al cuerpo para que reaccionen a lo que sucede a su alrededor".

- "Los seres vivos son diversos porque son capaces de captar diferente información y reaccionan de forma diversa"

- NOTA: se trabajo con algunos animales vertebrados. Otros seres vivos (animales) también tienen partes especiales para captar lo que sucede a su alrededor.

\section{Modelo intermediario 3, tercer y cuarto años}

- En nuestro cuerpo tenemos partes especiales para captar lo que sucede a nuestro alrededor: los órganos de los sentidos"

- "Cada parte especializada capta cosas diferentes: la luz (ojos), la temperatura y cuando te toca algo (piel y lengua), el sonido (oídos), el sabor (lengua) y el olor (nariz)".

- "Los ojos nos permiten ver, pero hay diferencia entre lo que se ve y lo que hay en el mundo"

- "la luz viaja en línea recta (modelo geométrico de la luz)"

- "el ojo funciona como una cámara obscura (analogía)"

- "Se forman imágenes en la parte trasera del ojo"

- "Los ojos se comunican con el cerebro por medio de cablecitos o nervios".

- "El cerebro junta las dos imágenes provenientes del ojo y forma una imagen en $3 \mathrm{~d}$ que nos permite saber qué hay a nuestro alrededor, la posición, distancia, profundidad, colores, etc. de los objetos"

- "El cerebro almacena las ideas y manda ordenes al cuerpo para que reaccionemos a lo que sucede a nuestro alrededor".

\section{Modelo intermediario 4, quinto y sexto años}

- "Los órganos de los sentidos captan información específica del medio"

- "Los órganos de los sentidos tienen pequeñas partes (células) especializadas para poder captar cierto estímulo"

- "Las partes o células especializadas se conectan con los nervios, que a su vez se conectan con el cerebro"

- "El cerebro recibe la información que viaja por los nervios y la traduce para que sepamos lo que pasa a neustro alrededor"

- "El cerebro tiene zonas especializadas para recibir y procesar cierto tipo de información"

\section{Modelo intermediario 5, secundaria}

- El sistema nervioso esta formado por diferentes partes, cada una tiene una función especifica.

- Hay estímulos externos (ruidos) e internos (hormonas).

- Algunos estímulos tienen efectos que ya no se pueden cambiar (irreversibles) como algunas drogas.

- Existen enfermedades del sistema nervioso, se deben a que alguna parte no esta realizando su función.

Cuadro 3. Modelos intermediarios propuestaos para el modelo "relación" en seres vivos. 
Un elemento importante es considerar que la introducción de ideas está muy relacionada de las actividades llevadas a cabo y el contexto de trabajo. Un punto clave del proceso de modelización es el hecho de que lo que se modeliza son los fenómenos. Es decir el modelo lo es en tanto permite la explicación de un fenómeno del mundo. Siendo así, podemos afirmar que en el cuadro 3 se presenta el modelo de forma parcial, es decir únicamente las ideas teóricas, pero no los fenómenos que explican, los cuales fueron observados en clase a través de experimentación, manipulación, simulación o analogías.

En el caso específico de la propuesta para tercero y cuarto año, se eligió uno de los sentidos para su estudio a profundidad, sin embargo es necesaria más investigación para determinar la pertinencia de ello y en su caso si el sentido de la vista sería el más adecuado para este nivel escolar.

\section{Comentarios finales}

Bajo una metodología de desarrollo curricular basado en la modelización en la que se consideran los aportes teóricos y la práctica en el aula, identificamos que los primeros nos facilitaron el generar el modelo blanco y con ello una plataforma de diseño. Sin embargo, fueron las aportaciones de los docentes y el análisis de las actividades en el aula quienes permitieron una comprensión emergente para puntualizar las ideas a desarrollar en cada modelo intermediario y complementar el modelo blanco. En ese sentido los modelos blanco e intermediarios no solo se conforman a través de la trasposición de los modelos eruditos de referencia o las ideas de partida de los alumnos, sino de las aportaciones docentes y del análisis de la práctica real en las escuelas.

Agradecimeintos: Esta investigación fue apoyada por el Consejo Nacional de Ciencia y Tecnología y la Secretaría de Educación Pública-Subsecretaría de Educación Básica, México, a través del proyecto 48374 . Se agradece el apoyo en la revisión del manuscrito a Yei Renteria.

\section{Bibliografía}

Akker, J.van den (2003). The science curriculum: between ideals and outcomes. En B. J. Fraser \& K. G. Tobin. International Handbook of Science Education (Part One). (pp. 421-447). Netherlands: Kluwer.

Archer, A., Arca, M. \& Sanmartí, N. (2007). Modeling as a Teaching Learning Process for Understanding Materials: A Case Study in Primary Education. Sci. Ed. 91:398-418.

Clement, J. (2000). Model based learning as a key research area for science education. Int. J. Sci. Educ. 22(9):1041-1053.

Coll, R. K., France, B. \& Taylor, I. (2005). The role of models and analogies in science education: implications from research. International Journal of Science Education, 27 (2): 183-198.

Fortus, D. \& Krajcik, J. (2012). Curriculum coherence and learning progressions. En B. J. Fraser; K. G. Tobin \& C. McRobbie. Second International Handbook of Science education. (Part Two). (pp. 783-798). NY: Springer.

García, J.E. (1997). La formulación de hipótesis de progresión para la construcción del conocimiento escolar: una propuesta de secuenciación de la enseñanza de la ecología. Alambique, 14: 37-48.

García, J.E. (1998). Hacía una teoría alternativa de los contenidos escolares. España: Diada.

García, P. (2005). Los modelos como organizadores del currículo en biología. Enseñanza de las Ciencias. Número extra. Pp. 1-5.

Giere, R.N. (Ed.). (1992).Cognitive models of science. USA: University of Minnesota Press.

Gómez,A., Sanmartí, N. \& Pujol, R. (2007). Fundamentación teórica y diseño de una unidad didáctica para construir el modelo de ser vivo en la escuela primaria. Enseñanza de las Ciencias, 25(3):325-340

Gómez, A. (2008). Construcción de explicaciones multimodales: ¿Qué aportan los diversos registros semióticos? Revista latinoamericana de estudios educativos, 4(2): 83 - 99.

Gómez, A. (2009a). Un análisis desde la cognición distribuida en preescolar: el uso de dibujos y maquetas en la construcción de explicaciones sobre órganos de los sentidos y sistema nervioso. Revista Mexicana de Investigación Educativa, 14(41): 403-430. 
Gómez, A. (2009b) El estudio de los seres vivos en la Educación Básica. Enseñanza del sistema nervioso desde un enfoque para la evolución de los modelos escolares. Universidad Autónoma de Nuevo León, México.

Gómez, A., Adúriz-Bravo, A., Guerra-Ramos, M. \& MarbàTallada, A. (2009). Explanations on sense organs and nervous system: A content analysis of primary school textbooks. En Memorias del congreso NARST. (pp. 1-12). Garden Grove. CA, USA.

Gómez, A. (2011). Desarrollo de modelos científicos escolares sobre órganos de los sentidos y sistema nervioso en educación básica. En: J.B. García Horta \& C. Campillo Toledano (Eds.), Escenarios y actores educativos: experiencias y reflexiones sobre la educación en México. (pp. 60-82). México: UANL.

Gómez, A. (2013). Explicaciones narrativas y modelización en la enseñanza de la biología. Enseñanza de las Ciencias. 31(1), 11-28.

Gutiérrez, R. (2004). La modelización y los procesos de enseñanza aprendizaje. Alambique 42, 8-18.

Izquierdo, M., Espinet, M., García, M. P., Pujol, R.M. \& Sanmartí, N. (1999). Caracterización y fundamentación de la ciencia escolar. Enseñanza de las Ciencias, núm. Extra, 79-91.

Izquierdo, M. y Adúriz-Bravo, A. (2003). Epistemological foundations of school science. Science \& Education, 12(1), 27-43.

Kress, G., Jewitt, C., Ogborn, J. \& Tsatsarelis, C. (2001). Multimodal teaching and learning. The rhetorics of the science classroom. London: Continuum.

Lockheed, Marlaine (1993) The condition of primary education in developing countries, En H. Levin \& M. Lockheed (Eds.) Effective schools in developing countries, London: World Bank.

Matthews, M. (2007). Models in science and science education: An introduction. Science \& Education, 16, 647-652.

Perales, F.J.; Sierra, J.L. \& Vilchez, M. (2002) ¿Innovar, investigar? ¿Qué hacemos en didáctica de las ciencias? Alambique, 34, 71-81

Sanmartí, N. (2002). Didáctica de las ciencias en la educación secundaria obligatoria. Madrid: Síntesis Educación.
Secretaría de Educación Pública (2008). Educación Básica. Primaria. Plan de Estudios 2009. Etapa de Prueba. México: SEP

Sensevy, G., Tiberghein, A., Sylvain-Laubé, J. \& Griggs, P. (2008). An epistemological approach to modeling: Cases studies and implications for science teaching. Science education, 92. 424-446. 